\title{
STUDY OF SUPERGRANULES
}

\author{
HAIMIN WANG and HAROLD ZIRIN
}

Big Bear Solar Observatory, California Institute of Technology, Pasadena, CA 91125, U.S.A.

(Received 22 June, 1988; in revised form 17 October, 1988)

\begin{abstract}
Results of a detailed study on supergranule lifetime and velocity fields are presented. We show the correlation between the observed downdraft velocity and the network magnetic flux elements on the quiet sun. After excluding areas with magnetic flux density $\geq 25 \mathrm{G}$, we find that the upper limit of the supergranule vertical speed is $0.1 \mathrm{~km} \mathrm{~s}^{-1}$ for both downdraft and updraft, and the r.m.s. speed is $0.03 \mathrm{~km} \mathrm{~s}^{-1}$. By observing the evolution of individual supergranules, we find that the average lifetime of supergranules might be $\geq 50$ hours. We describe different ways of formation and decay of supergranular cells. New cells usually form in an area containing no pre-existing supergranule velocity fields. Cells may disappear in two ways: fragmentation and fading away.
\end{abstract}

\section{Introduction}

The supergranulation velocity field was discovered by Hart $(1954,1956)$. It has been interpreted as a convective flow pattern (Leighton, Noyes, and Simon, 1962; Simon and Leighton, 1964; Leighton, 1964, 1969). The lifetime of supergranule cells is an important parameter with which the velocity and temperature structure within a cell may be estimated by convective theory (Antia, Chitre, and Pandey, 1981). The lifetime is important for another reason. Leighton $(1964,1969)$ attributed the observed breakup and dispersion of active region magnetic fields over the solar surface to a random walk process. If supergranules, which are responsible for moving the magnetic field, have a mean lifetime of about 20 hours, then the breakup of magnetic active regions may be explained by Leighton's mechanism.

Studies of supergranule lifetime by Simon and Leighton (1964), based on the crosscorrelation calculation of the chromospheric network in the $\mathrm{CaK}$ line, gave a lifetime of about 20 hours. Rogers' (1970) and Janssens' (1970) correlation analysis of 62 hours continuous $\mathrm{H} \alpha$ chromospheric network data from the Arctic showed a similar result. The investigations of Smithson (1973), Worden and Simon (1976), and Duvall (1980), however, found the lifetime to be about 40 hours. Livingston and Orrall (1974) noticed certain magnetic structures (magnetic pukas) persisting for 4 to 7 days. They explained the phenomenon as the existence of very stable supergranule convective cells. Kubicêla (1976) gave evidence that some supergranules have a lifetime of 72 hours or more in the quiet Sun.

The amplitude of the horizontal component of supergranule velocity has been well studied. Its peak speed has been found to be around 0.3 to $0.5 \mathrm{~km} \mathrm{~s}^{-1}$ (Leighton, Noyes, and Simon, 1962; Worden and Simon, 1976). However, despite numerous studies, the amplitude of the vertical velocity in supergranules is still unclear. Different authors give very different r.m.s. speeds ranging from on the order of $0.01 \mathrm{~km} \mathrm{~s}^{-1}$ (Musman and Rust, 1970; Giovanelli, 1980) to about $0.4 \mathrm{~km} \mathrm{~s}^{-1}$ (Skumanich, Smythe, and Frazier, 
1975; Deubner, 1971). The correlation between downdraft velocity and magnetic flux density is well known (e.g., Frazier, 1970). Studies on the vertical velocity were reviewed and studied further by Giovanelli (1980). His result shows that supergranule vertical velocities have r.m.s. values $\leq 0.01 \mathrm{~km} \mathrm{~s}^{-1}$. Downward velocity $\geq 0.1 \mathrm{~km} \mathrm{~s}^{-1}$ in regions of strong magnetic fields is attributed to motion inside the elements themselves that is unrelated to supergranule motion.

In this paper, we study the vertical velocity of supergranules and its relationship with the magnetic flux density. We also measure the mean lifetime of supergranule cells by direct study of the evolution of the horizontal component of supergranular velocity fields.

\section{Observation}

Some of the velocity observations presented here were made with the 512 channel magnetograph (Livingston et al., 1976a) at the Solar Vacuum Telescope of the National Solar Observatory, Kitt Peak (Livingston et al., 1976b). The velocity is inferred from the difference in intensities in the two wings of the Fe I line ( $8688 \AA)$. Magnetograms were obtained at Kitt Peak and Big Bear Solar Observatory (BBSO) simultaneously with the velocity observations. The pixel size in the Kitt Peak data is $1^{\prime \prime}$ and the scan area is $256^{\prime \prime} \times 256^{\prime \prime}$ for the October 1985 run, and $400^{\prime \prime} \times 256^{\prime \prime}$ for the May 1986 run. To remove the five-min oscillation most efficiently, the time interval between images was set at $150 \mathrm{~s}$.

Additional velocity and magnetic field observations were made with the videomagnetograph (VMG) system at BBSO during the summers of 1985 and 1986. While the Kitt Peak magnetograph scans the area line by line with the spectrum slit, at Big Bear, a narrow bandpass Zeiss filter provides two dimensional images instantly. The VMG system at Big Bear was described in detail by Mosher (1976a) and Zirin (1985). CaI $6103 \AA$ was used and the VMG and Doppler modes were alternated for the magnetic field and velocity field observations. The pixel size is about $0.5^{\prime \prime}$, the area covered is $250^{\prime \prime} \times 250^{\prime \prime}$. The duration of each observing day was $7-12$ hours and the same regions were usually followed for three consecutive days.

The vertical velocity of supergranules may be observed only in the solar disk center, while the horizontal velocity can be clearly observed only when the heliocentric angle is larger than $20^{\circ}$. Thus targets were set at the disk center to study the vertical component of the supergranule velocity, and away from the disk center 27 to $55^{\circ}$ to study the horizontal component.

\section{Data Reduction}

The calibration of magnetic and velocity fields was done by using the solar rotation as the calibrator (Shi, Wang, and Patterson, 1986). The solar rotation and non-uniformity in the images were removed by subtracting a two-dimensonal smoothed background image. The five-minute oscillation was removed by averaging 60 images spanning 1 hour. 
Since the various velocity phenomena (granulation, horizontal and vertical velocity of supergranulation and five-min oscillation) have different spatial scales, the information on each of these velocity fields can be separated within the spatial frequency domain of the Fourier transform. Thus, to isolate the horizontal component of supergranular velocity fields, the data are Fourier transformed, and all frequencies representing size scales significantly different from the supergranular scale $(32000 \mathrm{~km})$ are removed, then the transform is inverted to produce a filtered picture.

Dopplergram movies were made from the time sequence observation using the Megavision Image Processor system at Caltech. The movies greatly facilitate the detailed study of the evolution of supergranule velocity fields.

\section{Vertical Velocity}

To study the vertical component of supergranule velocity fields, we averaged 60 center disk Dopplergrams taken at a rate of 1 image $\min ^{-1}$. The observations were made on August 21, 1985 and August 17, 1987 at BBSO. Only data with the best seeing were used. On August 17, 1987 several magnetograms were taken immediately before and following the one hour Dopplergram observation.

Figure 1(a) shows a 60-frame averaged Dopplergram on August 21, 1985. A histogram of the distribution of vertical speed for the Dopplergram shown in Figure 1(a) is plotted

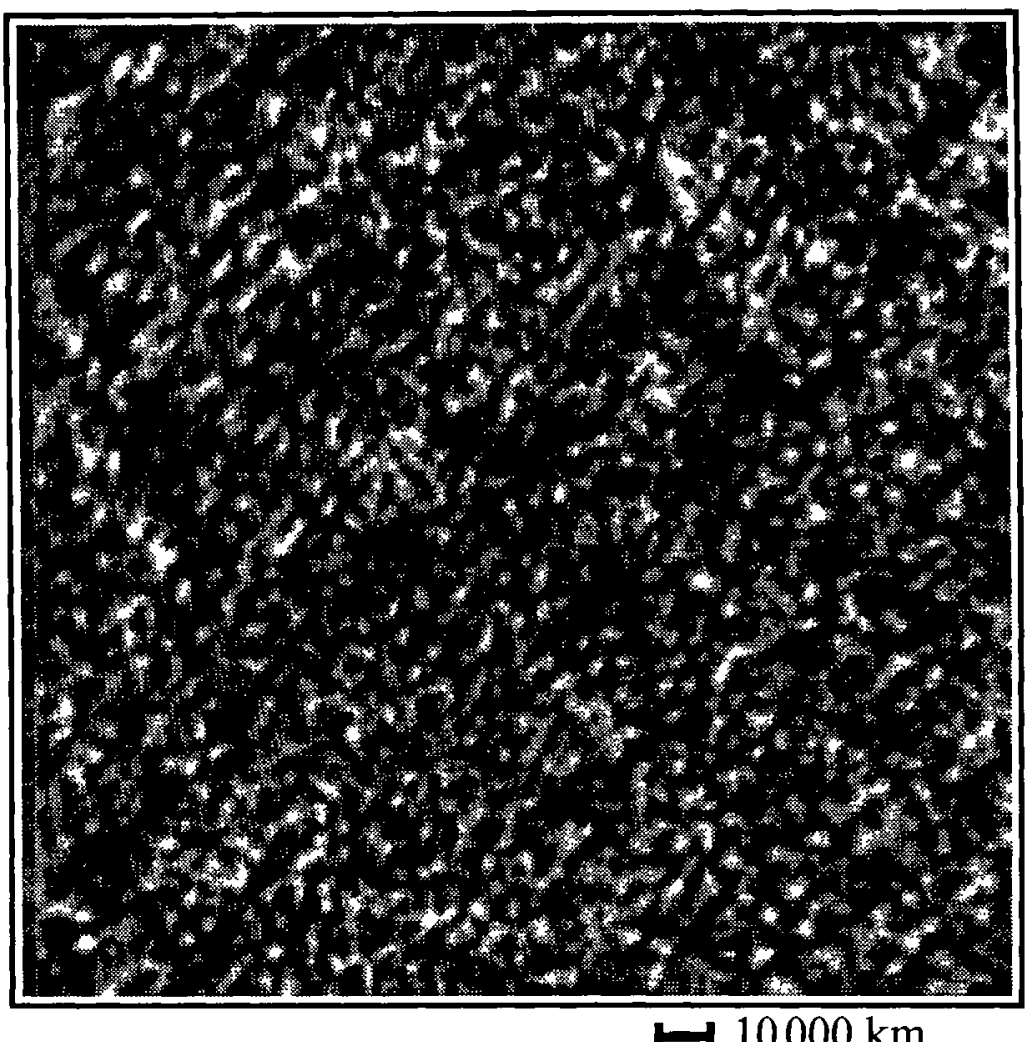

Fig. 1a. A Dopplergram made on August 21, 1985, after one-hour averaging and two-dimensional smoothing. 


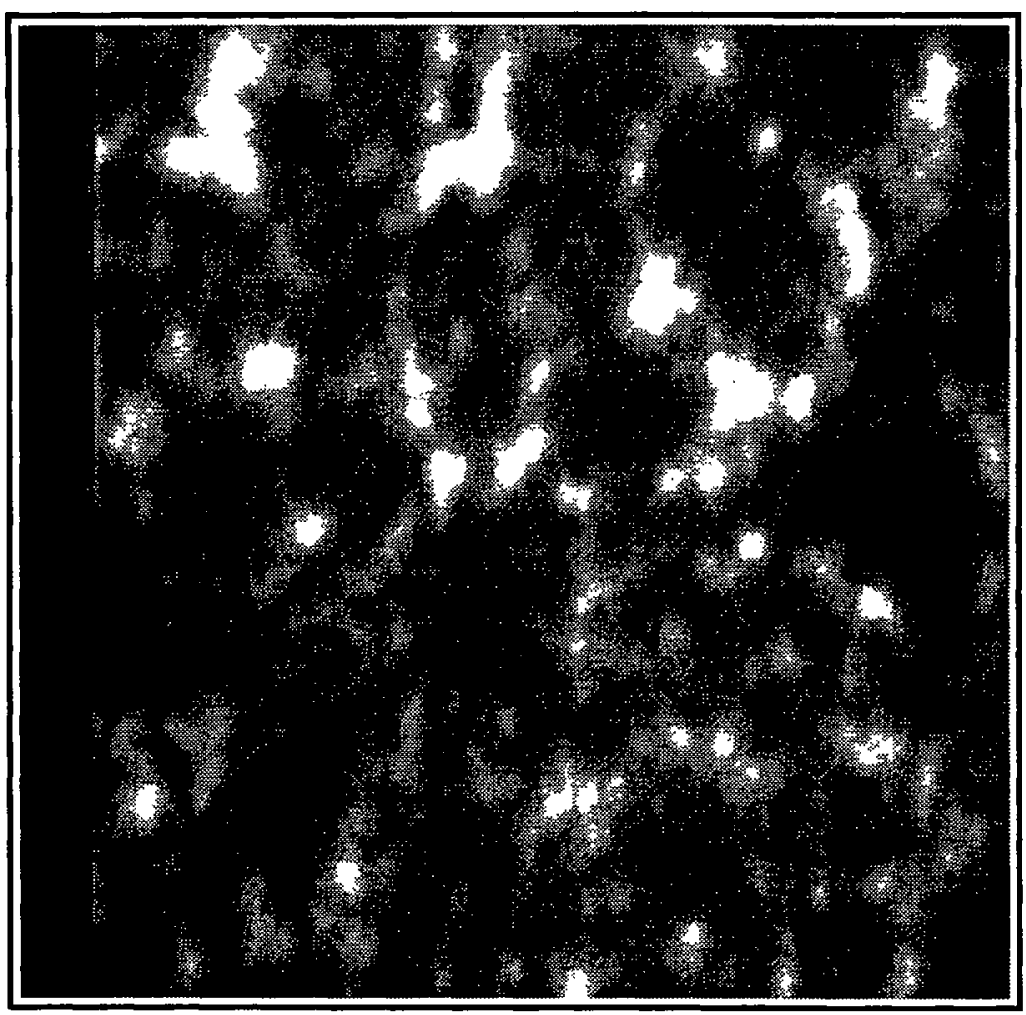

Fig. 1b. An averaged $\mathrm{H} \alpha-0.1 \AA$ negative image corresponding to Figure 1(a).

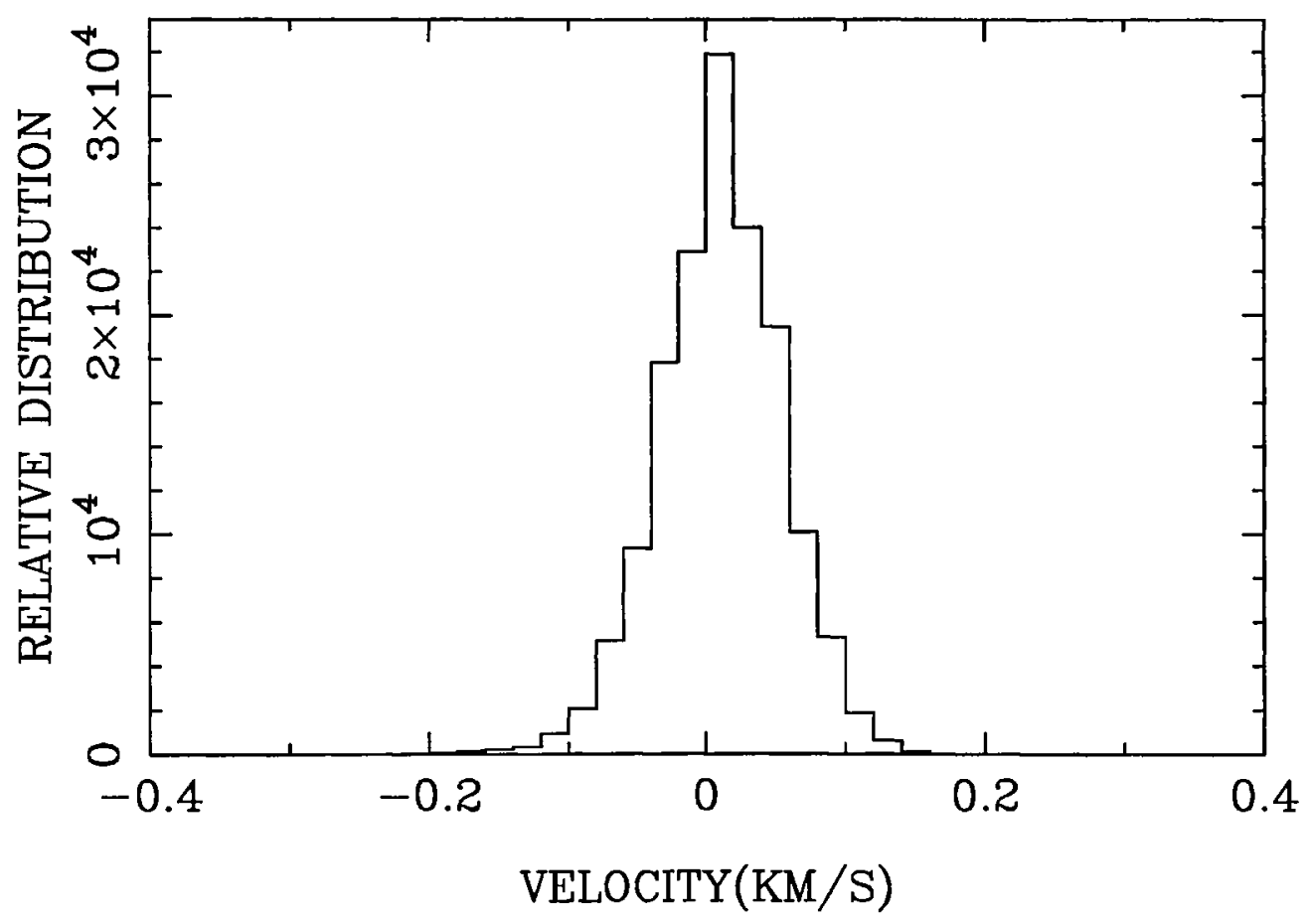

Fig. 2. A histogram to show the distribution of the vertical velocity fields for the Dopplergram shown in Figure 1(a). 
in Figure 2. It shows that $98.4 \%$ of the image area is covered by pixels with velocity $\leq 0.1 \mathrm{~km} \mathrm{~s}^{-1}$. The r.m.s. velocity is $0.04 \mathrm{~km} \mathrm{~s}^{-1}$. There is a small tail on either side of the histogram representing higher velocity regions. The blue shift tail does not exceed $0.15 \mathrm{~km} \mathrm{~s}^{-1}$ and red shift tail does not exceed $0.20 \mathrm{~km} \mathrm{~s}^{-1}$. Many dark patches in Figure 1(a) represent isolated areas of downward motion probably corresponding to network magnetic elements, but there were no corresponding magnetograms available to confirm this. However, simultaneous $\mathrm{H} \alpha$ off band data were obtained. Figure 1(b) shows a negative print of a $\mathrm{H} \alpha-0.5 \AA$ filtergram averaged over 60 images spanning the same one-hour time interval as Figure 1(a). The correlation coefficient between two images is 0.55 . Roughly, the downdraft velocity fields coincide with the $\mathrm{H} \alpha$ network.

To study the relationship between the downdraft and magnetic flux density, we analyzed a set of Dopplergrams and their corresponding magnetograms made on August 17, 1987. Figure 3(a) shows a Dopplergram averaged over an hour and Figure 3(b) shows the corresponding magnetogram. The r.m.s. velocity of the Dopplergram shown in Figure 3(a) is $0.038 \mathrm{~km} \mathrm{~s}^{-1}$. All magnetic elements show downdraft, but there are some downdrafts that do not correspond to magnetic fields.

In Figure 4(a), we plot the average vertical velocity vs the magnetic flux density for the data shown in Figures 3(a) and 3(b). For $B=0$ to $B=50 \mathrm{G}$, a linear least-square fit gives

$$
V\left(\mathrm{~km} \mathrm{~s}^{-1}\right)=-1.2 \times 10^{-3} B(\mathrm{G}) .
$$

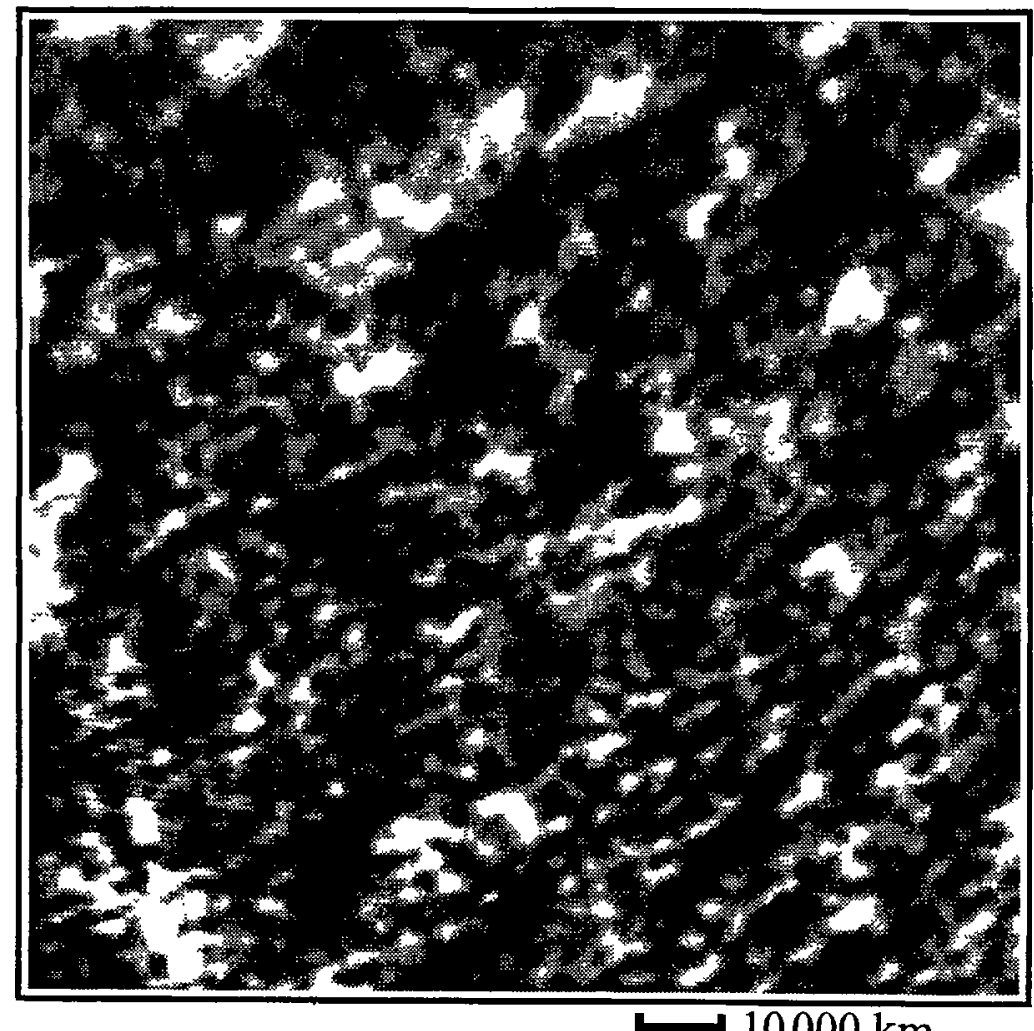

Fig. 3a. A Dopplergram made on August 17, 1987, after one-hour average and 2-D smoothing. It is observed at the disk center. 


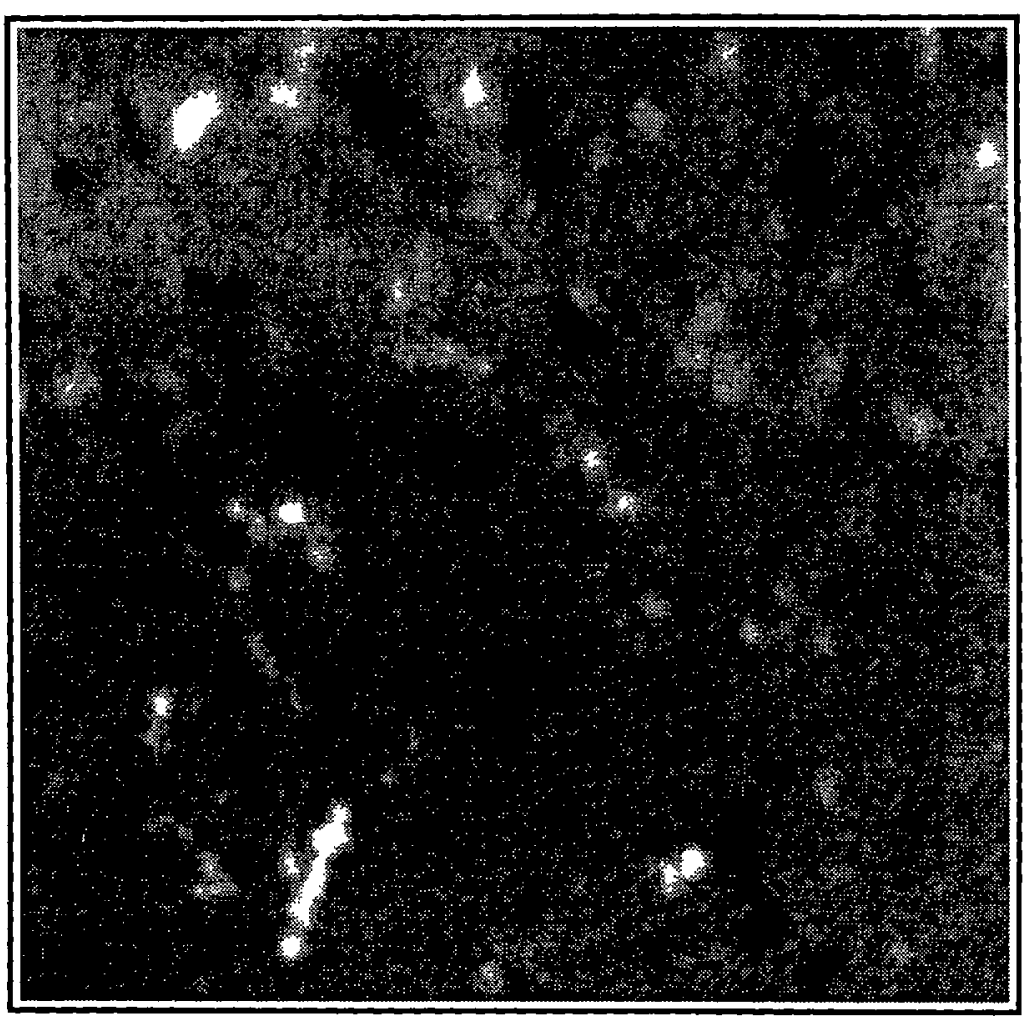

Fig. 3b. A magnetogram corresponding to Figure 3(a).

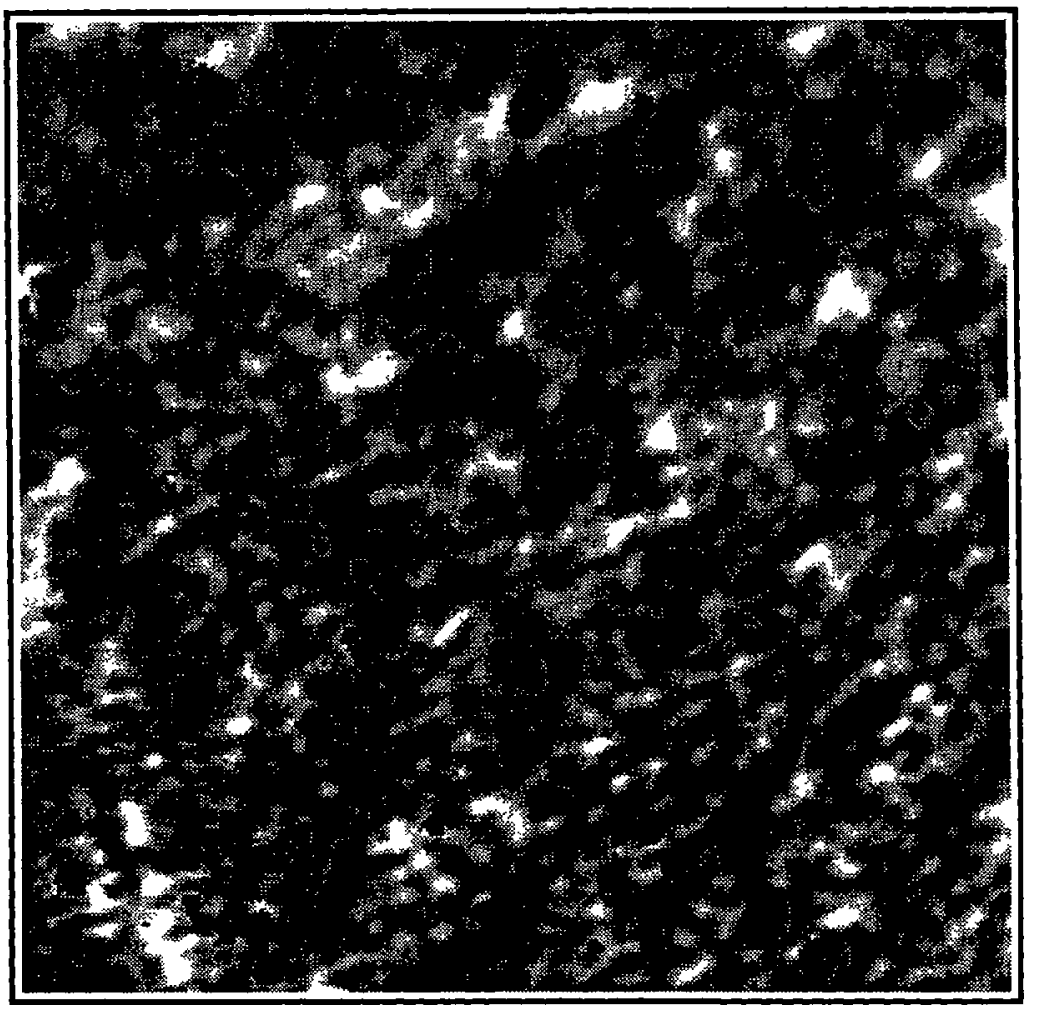

Fig. 3c. The Dopplergram after blocking areas with $B \geq 25 \mathrm{G}$. 


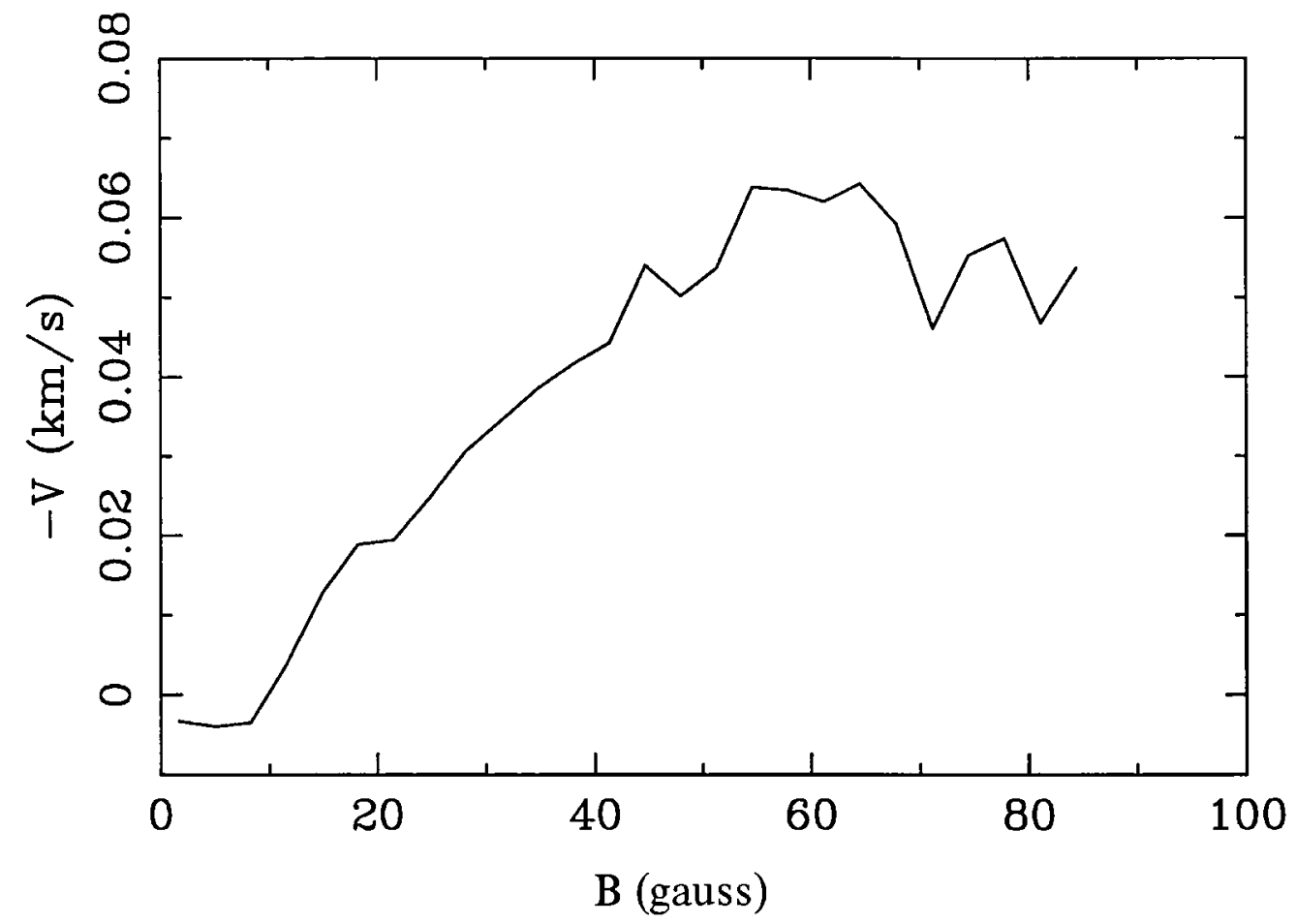

Fig. 4a. Average vertical velocity vs. magnetic flux density for the data shown in Figures 3(a) and 3(b). The bin size is $2 \mathrm{G}$.

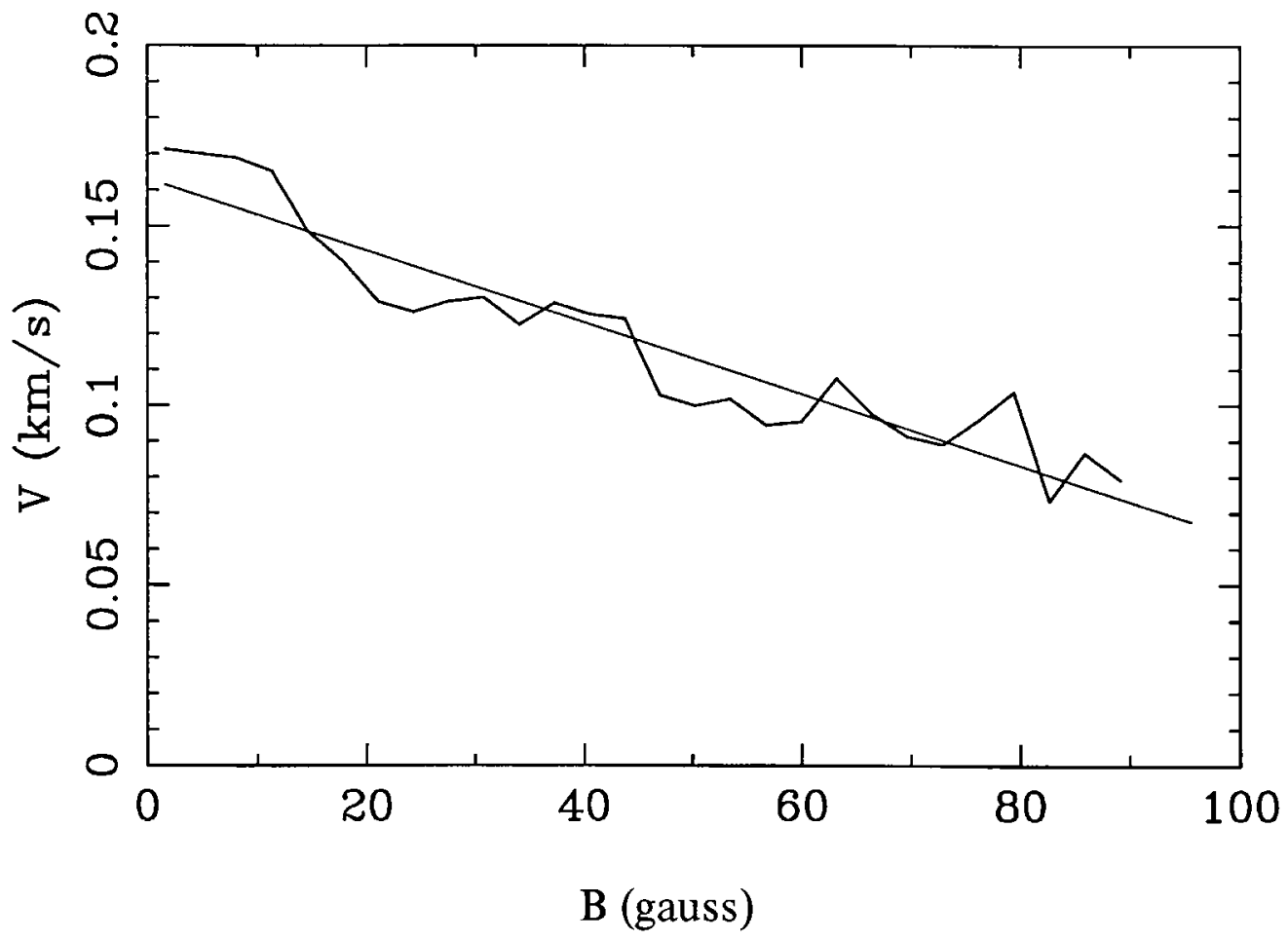

Fig. 4b. Average line-of-sight velocity vs magnetic flux density. The observation was made on May 11, 1986. The target was $40^{\circ}$ off the disk center, so that the velocity amplitude represents the horizontal component of the supergranule velocity. 
In this regime, the slope is consistent with the work by Chou et al. (1989). For $B$ greater than $50 \mathrm{G}$, there are less than 100 sampling points for each bin, so reliable data are restricted to $B \leq 50 \mathrm{G}$. One uncertain factor in our observation is the actual physical size of the downdraft velocity elements. If the elements are concentrated in a small area, the high downdraft velocity would be missed by our observation.

Following the method used by Giovanelli (1980), we block the areas in the Dopplergram in which the magnetic flux density exceeds $25 \mathrm{G}$. The blocked Dopplergram is shown in Figure 3(c). There are still many dark patches left in this image. The r.m.s. velocity of the blocked Dopplergram is $0.029 \mathrm{~km} \mathrm{~s}^{-1}$. This is 3 times the upper limit given by Giovanelli.

November, Toomre, and Gebbie (1981) and November et al. (1982) observed a convective mode which has a scale between the granulation and the supergranulation. They named this convective mode mesogranulation. The data they used are similar to the Dopplergrams we used to study the supergranule vertical velocity component. They found that the r.m.s. speed of mesogranules is $0.06 \mathrm{~km} \mathrm{~s}^{-1}$. Even if our observed r.m.s. vertical speed is totally contributed by mesogranules, our value is only about the half of what November et al. found.

There is an uncertain factor related to the results above. We used Ca $6103 \AA$ ( $g=1.5)$ which is a magnetically sensitive line to measure the Doppler signal. Magnetic signal may become manifest in the velocity if the field is strong (Worden and Simon, 1976). Mapping of circular Stokes's $V$ intensity into linear $Q$ or $U$ by some optics in the system will modulate against the entrance polarizer of the filter to produce a spurious Doppler signal. This effect might produce both red and blue shifts. In our observation we find only downdrafts (red shift) corresponding to the magnetic elements. Our VMG is a straight through system, the light goes through a refractor and then the filter, there is no oblique mirror. So system polarization is smaller than we can measure. Furthermore, recent velocity measurement on strong plage regions do not show such an effect and show velocities unrelated to magnetic fields (Zirin, 1989). We feel that the effect of Zeeman contamination by the polarization of the instrument is not an important factor.

Stenflo et al. (1984) used the Fourier Transform Spectrometer at Kitt Peak National Observatory to study the diagnostics of magnetic flux tubes. They concluded that if the Stokes's $V$ profiles had not been fully spectrally resolved, the $V$ asymmetry would have caused an apparent redshift of the $V$ profile leading to fictitious downdrafts in the network fluxtubes. But there is no quantitative discuss of this effect, and we cannot estimate what we should expect in our data. However, the $V$ asymmetry could not explain the downdrafts we find outside the magnetic elements. Furthermore, this asymmetry is important only when the magnetic flux is concentrated in small flux tubes with high flux density. So far, no direct evidence can prove that the flux is concentrated in the small size flux tubes.

In Figure 4(b) we plot the supergranule horizontal velocity amplitude vs the flux density for a region at a heliocentric angle of $40^{\circ}$. The horizontal velocity is smallest where the average magnetic flux density is greatest - average velocity decreases monotonically with flux density. This is consistent with the result that the strong 
magnetic elements are located at the boundaries of supergranules (Wang and Zirin, 1988).

\section{Lifetime of Supergranules}

\subsection{AUTO-CORRELATION OF DOPPLERGRAMS}

From the auto-correlation curves of Dopplergrams, Leighton, Noyes, and Simon (1962) and Simon and Leighton (1964) derived an average supergranule scale of $32000 \mathrm{~km}$. Averaging 8-day results, we get an average supergranule scale of $31200 \pm 2300 \mathrm{~km}$ by using the auto-correlation technique. Our analysis confirms Leighton, Noyes, and Simon's early results and ensures that our data contain the real supergranule signal.

\subsection{CORRELATION LiFETIME}

The lifetime of supergranulation cells have been measured using the cross-correlation function (Simon and Leighton, 1964; Worden and Simon, 1976). The mean lifetime is defined as the time needed for the cross-correlation coefficient to fall to $1 / e$. Figure 5 shows cross-correlation coefficients vs time and the linear fit for the data observed on October 13, 1985. The correlation lifetime derived from the linear fit is 18 hours. Figure 6 shows the result for three days of Doppler data, October 13-15, 1985r.An exponential least-squares fit gives a lifetime of 23 hours.

The correlation lifetime of magnetograms in the quiet region is about 10 hours (Wang, 1988), shorter than that derived from the correlation curve of Dopplergrams. This

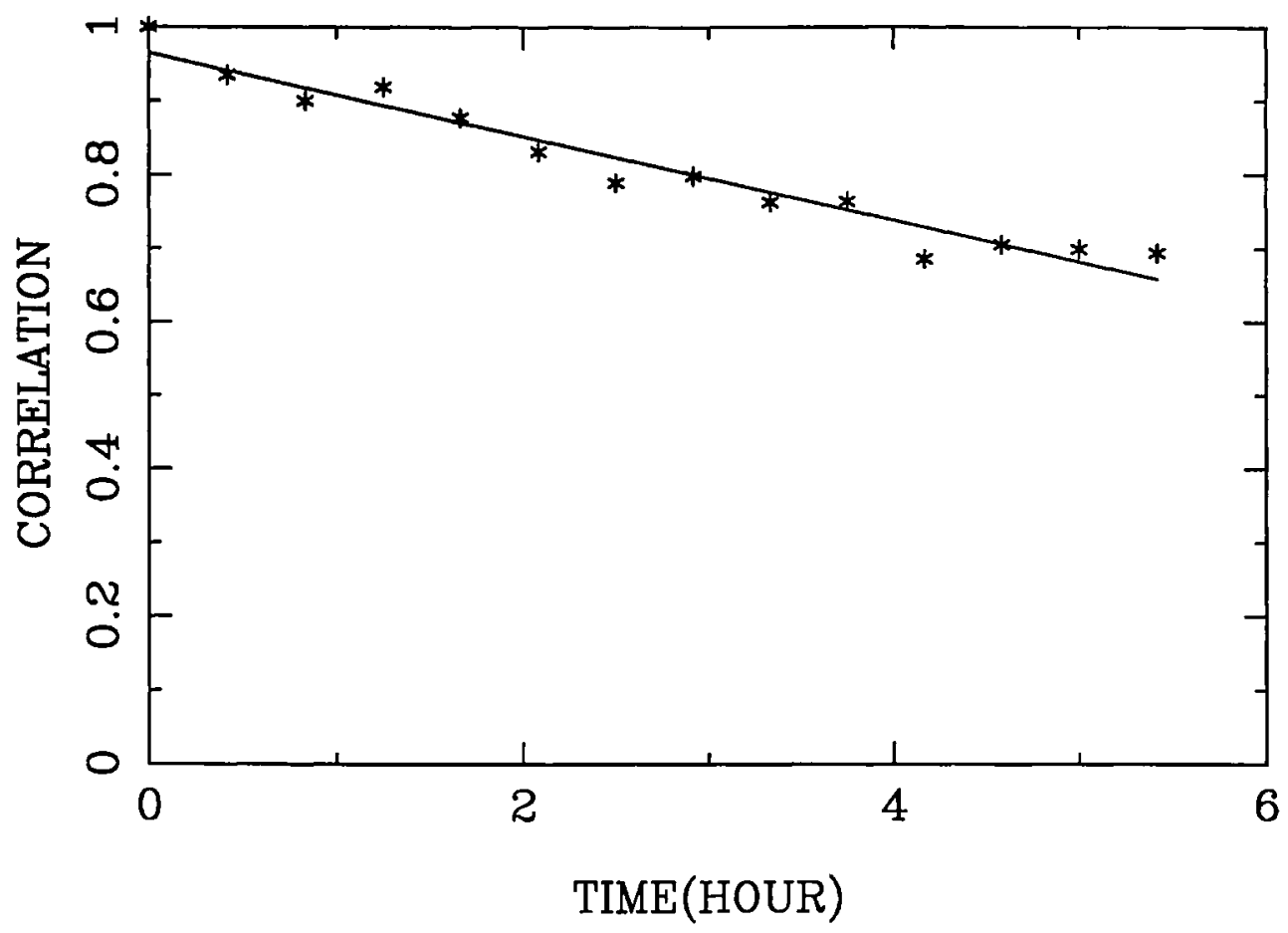

Fig. 5. The cross-correlation curve of the Dopplergrams made on October 13, 1985. 


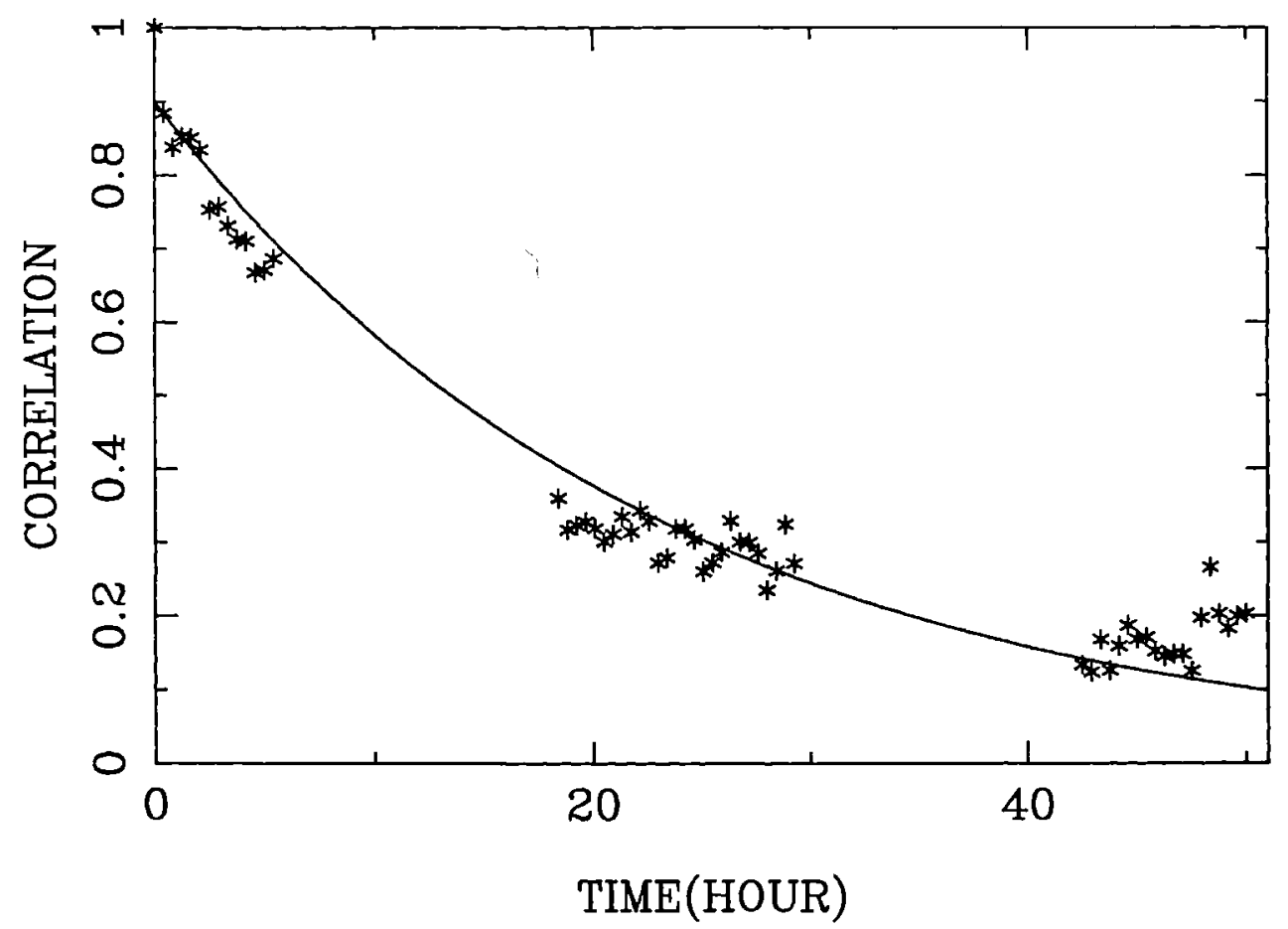

Fig. 6. The cross-correlation curve for combined 3-day observations, from October 13 to ctober 15, 1985.

confirms what Smithson (1973) suggested: the shorter supergranular lifetime derived from network studies was misleading due to the manner in which it was derived. Since the network is a 'thin' system defining only the cell boundary, not the entire cell, small changes in the shape of a cell can cause a large decrease in cross-correlation coefficients. This may produce a spurious short lifetime compared to the correlation lifetime derived directly from the velocity data.

\subsection{THE LIFETIME OF SUPERGRANULATION DERIVED FROM THE EVOLUTION OF SUPERGRANULES}

Does the correlation lifetime represent the real mean lifetime of supergranules? This is a problem we will discuss in this section.

The study of solar granules by Zirin and Wang (1987) shows that the average lifetime of granules is 16 minutes when measured visually, which is twice the value found by many authors using the correlation technique (e.g., Leigton, 1957).

One striking feature shown in supergranule movies is that the basic patterns of the supergranule velocity hardly change during one observing day. In an area of $256^{\prime \prime} \times 256^{\prime \prime}$, there are approximately 30 supergranular cells. If the mean lifetime equals the correlation time, which is about 24 hours as found by us and other authors, then in a 7-10 hour observation, $\frac{1}{3}$, or 10 , of cells should change substantially and lose their identity at the end of the observation. We found, however, in 8 days' supergranule movies we examined, it is very hard to find disappearance of more than 3 cells during any observing day. 
We express the mean lifetime of supergranules, $\tau$, as

$$
N(t)=N_{0} \exp (-t / \tau),
$$

where $N_{0}$ is the number of supergranule cells at the beginning of an observation, $N(t)$ is the number of cells remaining after time $t$. Figure 7 plots the number of supergranule cells that survived vs. time during the period of October 13 to 15,1985 . The least-squares fit to Equation (2) gives an average lifetime of 53 hours. We used the same data set to plot Figures 6 and 7.

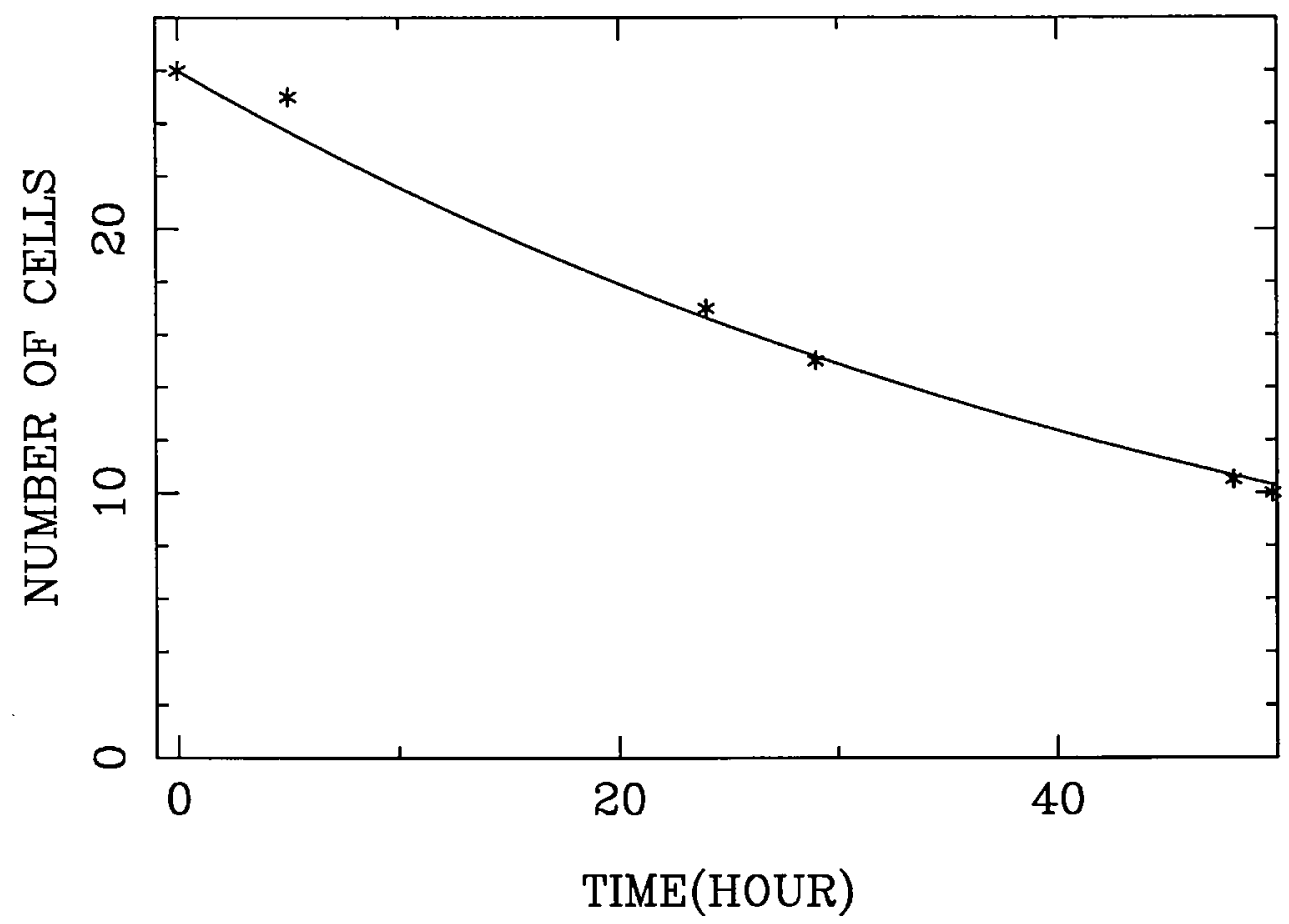

Fig. 7. The number of supergranule cells survived as a function of time, for the period from October 13 to October 15, 1985. The least-square fit gives a mean supergranule lifetime of 53 hours.

We analyzed five other sets of Doppler data, one observing day for each. Applying Equation (2) to these data, we obtained the lifetimes of 50 to 80 hours. We suggest that the average lifetime of supergranules is $\geq 50$ hours. The big difference between the correlation lifetime and the lifetime $\tau$ defined in Equation (2) may be due to the fact that it is possible for a cell to change its shape without losing its identity. The 50-hour value was also obtained by Zirin (1987) by tracking network elements.

\section{Evolution of Supergranules}

The use of supergranule Doppler movies is helpful in studying the short term evolution of supergranules. Unfortunately, since the average lifetime of supergranules is longer than 50 hours, a movie covering 7-10 hours of observation does not show long-term evolution. However, a few cases of growth and decay of supergranule cells are observed. Some examples are shown in Figure 8. It appears that most of the new cells form in a 


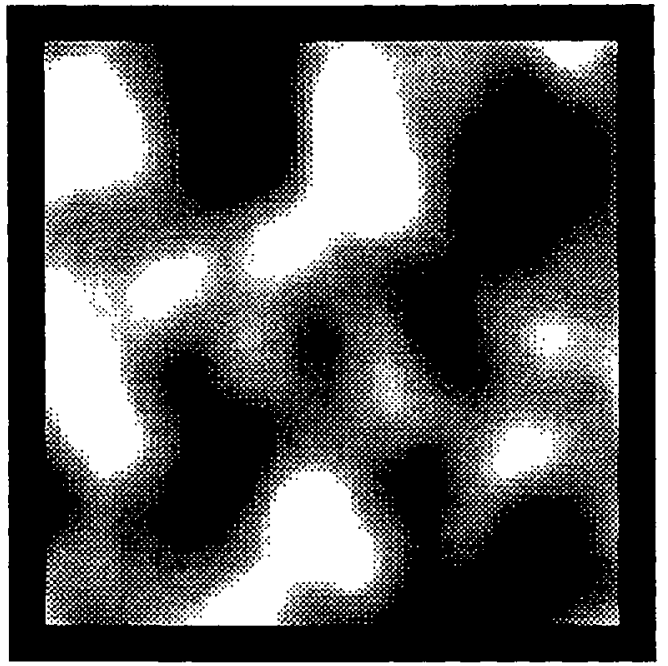

1659

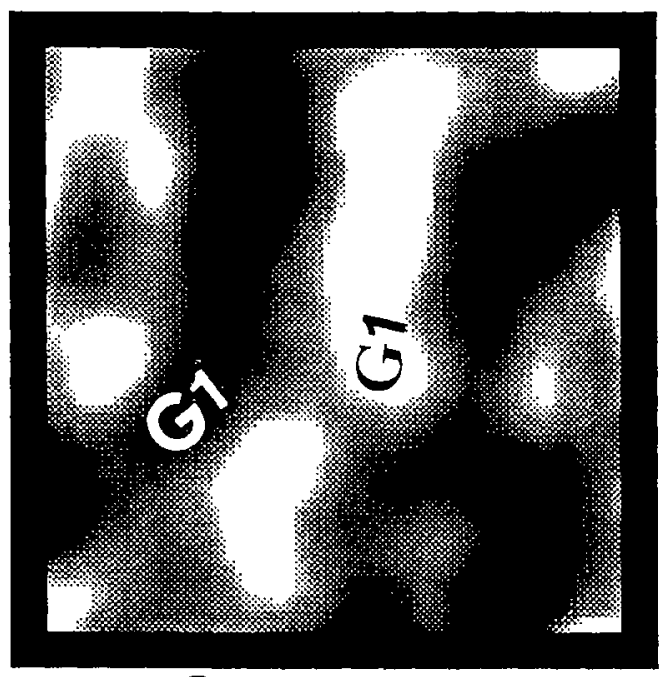

2238

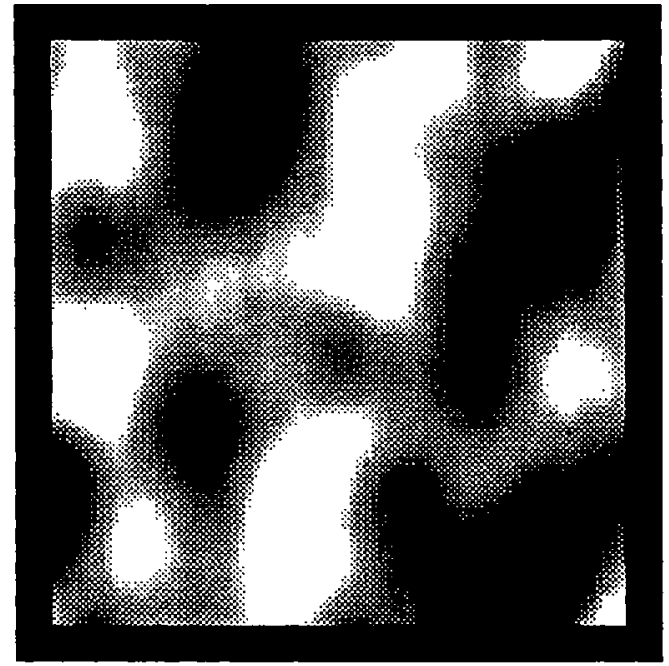

1955

$10000 \mathrm{~km}$

Fig. 8a.

blank area, containing no pre-existing velocity fields. Figure 8(a) shows an example of growing cell. The new cell is labeled as G1. The cell grows to a size of the normal supergranule in less than two hours. A cell may disappear by either fading away or by fragmentation. Figures $8(\mathrm{~b})$ and $8(\mathrm{c})$ show two examples of decaying cells. In Figure $8(\mathrm{~b})$, there are several features, labeled D1 through D3, that faded away. They left a blank space at the end of the observation. Figure 8(c) shows a case of cell fragmentation. A white velocity component (F1) fragmented first, then all the fragments faded away. A black element (F2), which belongs to another cell, starts to fragment at 22:00 UT. We did not find a single case in which a cell formed and disappeared during a 7-10 hour period. Our observation suggests that the supergranules have relatively short growth and decay phases of about 3 to 6 hours for each, and a long mature phase. However, the sampling from our 8 days of data is small, and the results are not statistically significant. 


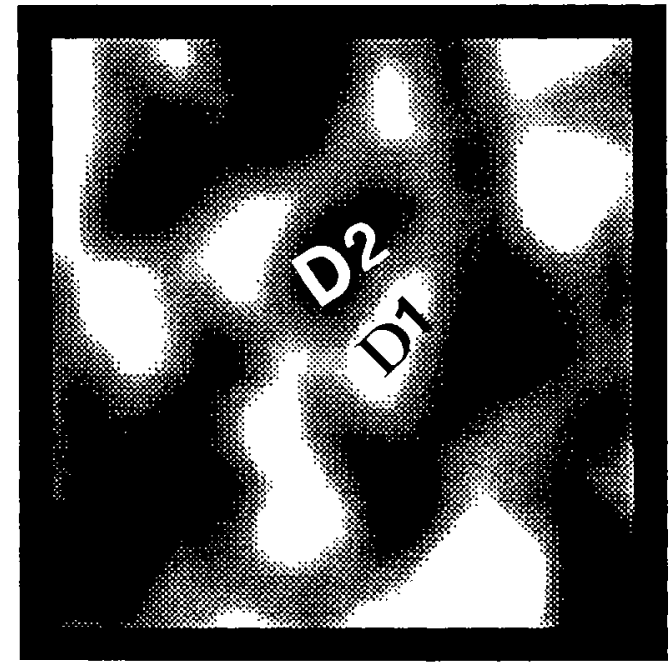

1659

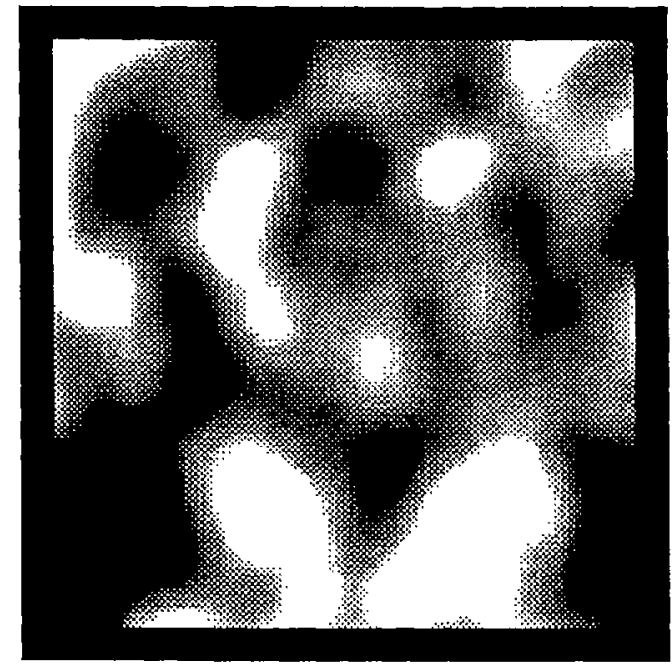

2238

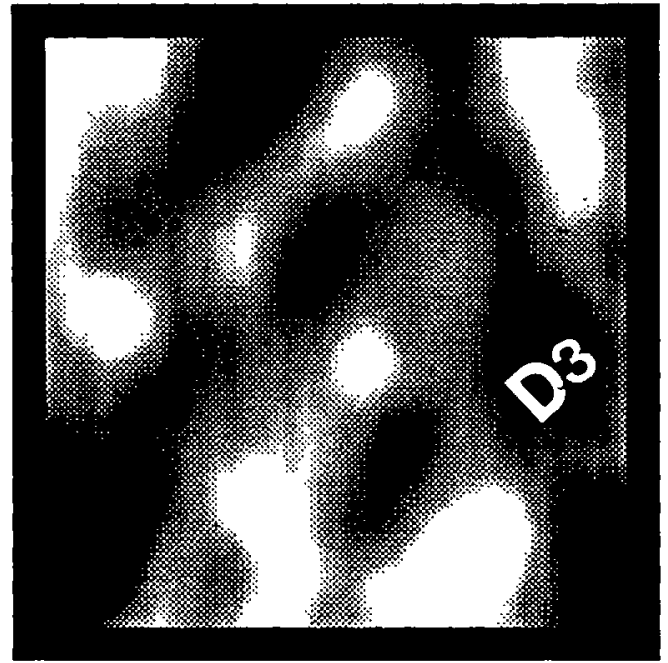

1955

Fig. 8b.

To study the evolution of the magnetic network during the process of appearing and disappearing supergranular velocity fields, superposed magnetograms and Dopplergrams are used. In a few cases the network expands when the velocity cell is growing. An example is shown in Figure 9(a): while a new cell (G2) is forming, it pushes a magnetic element of positive polarity to the right. However, the network does not appear to contract when the cell is decaying. The network magnetic elements of an old cell may become the elements of new cells. We give an example of a decaying velocity cell with its network fields, labeled D4, in Figure 9(b).

\section{Conclusion and Discussion}

From 8 days of observations made at Big Bear and Kitt Peak, we conclude that the mean lifetime of supergranules is much longer than 20 hours. Our results show that this 


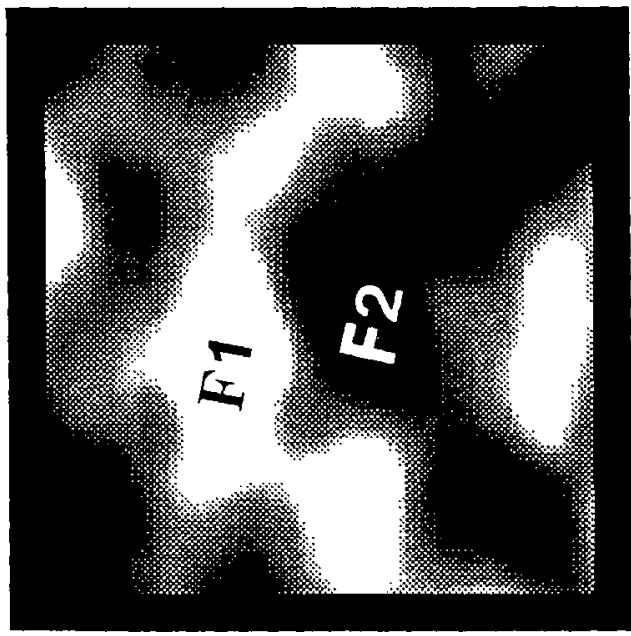

1659

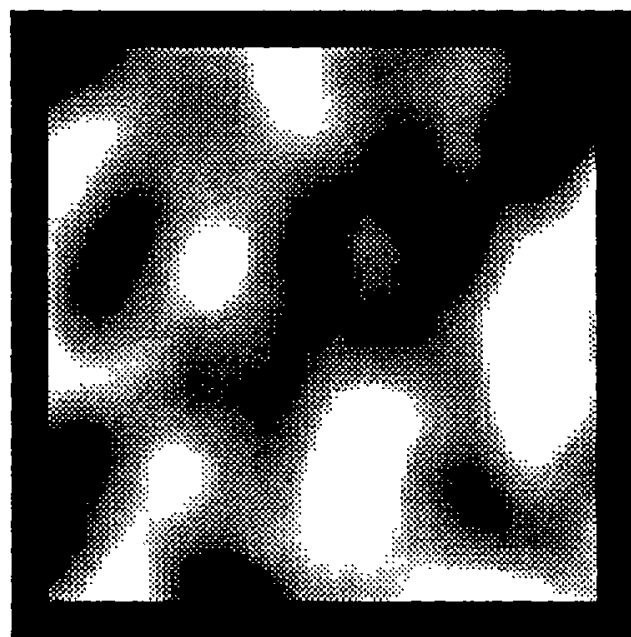

2238

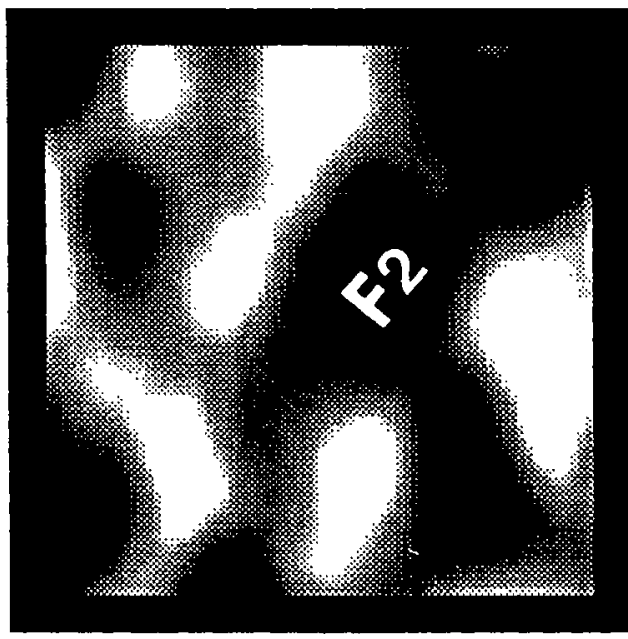

1955

Fig. 8. Several examples of the supergranule evolution. Data were obtained on May 13, 1986, UT time is given below each frame. (a) A new cell forms. (b) A cell fades away. (c) A cell disappears by fragmentation. All pictures have the same scale as marked in Figure 8(a). White is receding motion, and black, approaching. Solar limb is toward the right of the image. A supergranule cell is represented by a structure which has a white element in the right and a black one in the left.

lifetime is $\geq 50$ hours. The shape change of supergranules may reduce the crosscorrelation coefficient leading to a spurious short correlation lifetime. This conclusion is consistent with the study of the evolution of the magnetic network by Zirin (1987) and Martin (private communication). A longer lifetime of supergranules means a slower diffusion of solar magnetic fields (Worden and Simon, 1976). This implies that other mechanisms such as meridional flow (Mosher, 1976b) must share a larger burden of transporting the magnetic flux.

It was generally believed that the network originates from the residuals of active regions. Our results do not contradict this view. However, the network is continuously cancelled and replenished by both ephemeral regions and intranetwork (IN) magnetic 


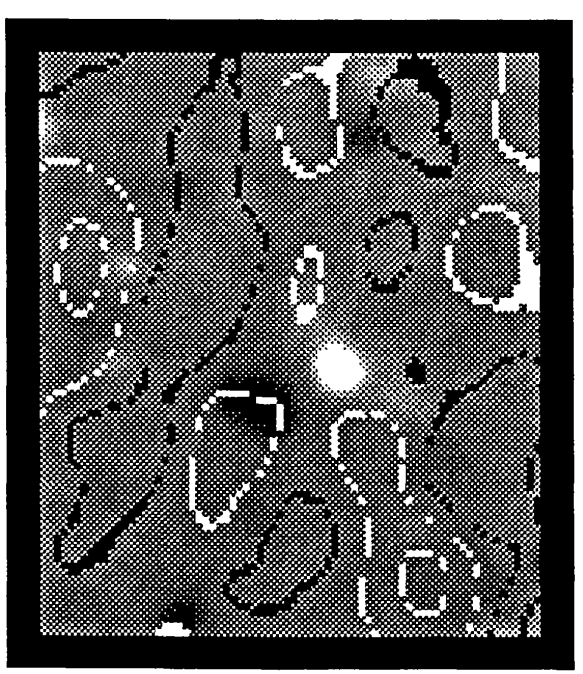

1948

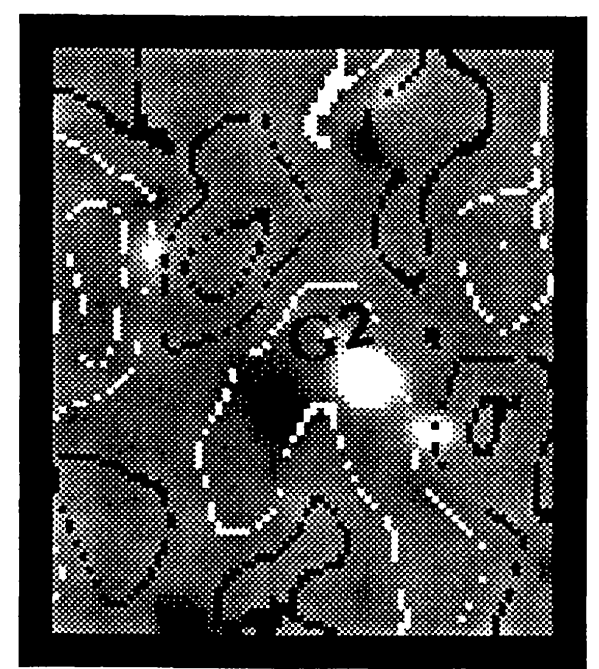

2312

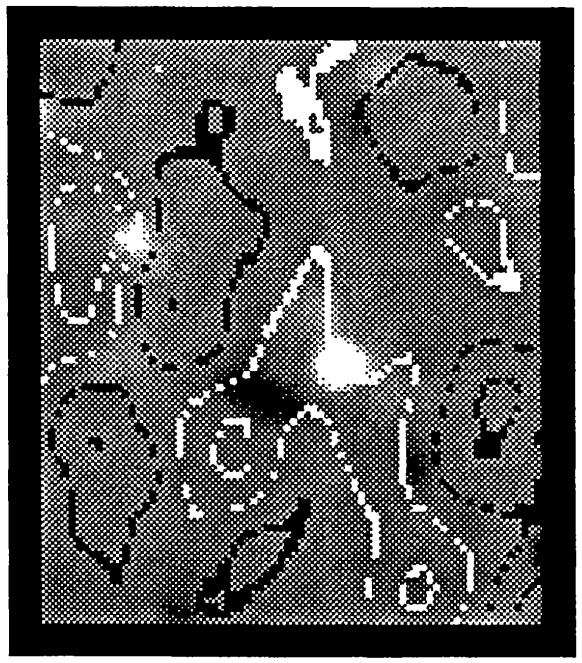

2103

Fig. 9a.

fields. Although the IN fields move under the control of the supergranule velocity fields (Martin, 1988; Wang and Zirin, 1988), since they have mixed polarities, the net flux accumulated from such a large amount of mixed polarity flux is small. In our observations of mixed polarity quiet regions, we have not observed that the supergranule velocity fields accumulate sufficient IN flux of the same polarity to form the network.

An upper limit for supergranule vertical velocity is found to be $0.1 \mathrm{~km} \mathrm{~s}^{-1}$. The linear relationship between the downdraft velocity and the magnetic flux density is good when the flux density is less than $50 \mathrm{G}$. There are a few questions that remain unanswered. (1) Is the downdraft real or it is due to the $V$ asymmetry, as discussed by Stenflo et al. (1984)? (2) What is the filling factor of the downdraft elements? (3) Do the downdraft areas outside magnetic elements represent real supergranule downdraft velocity? 


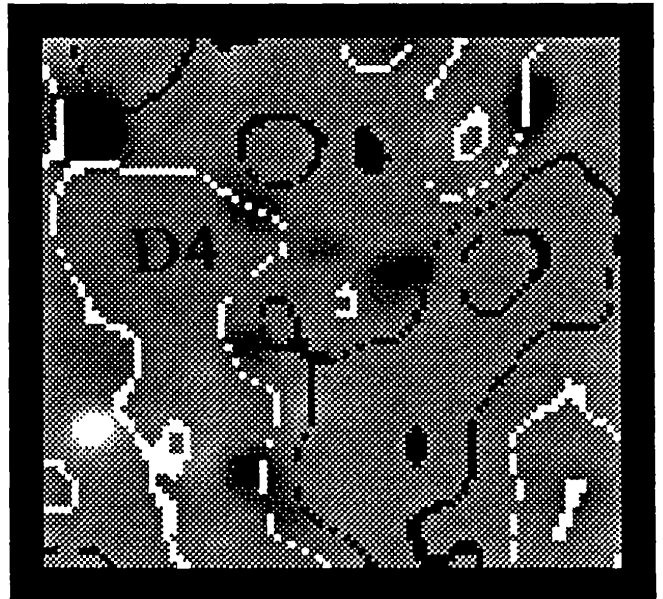

\section{8}

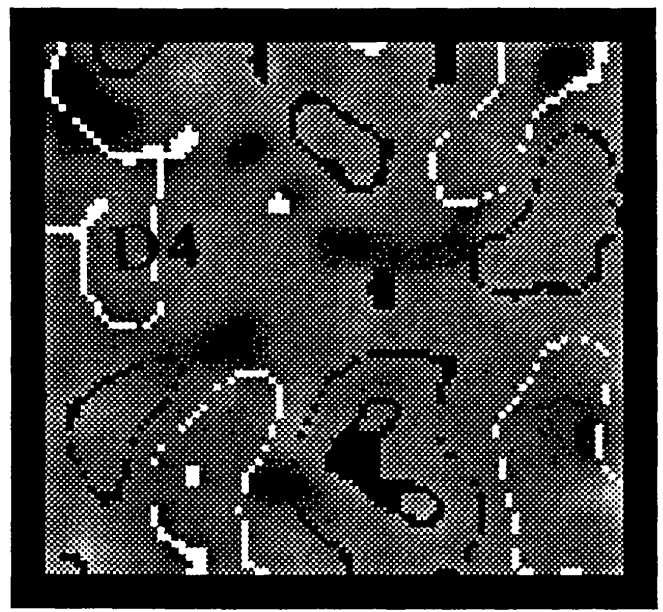

2312

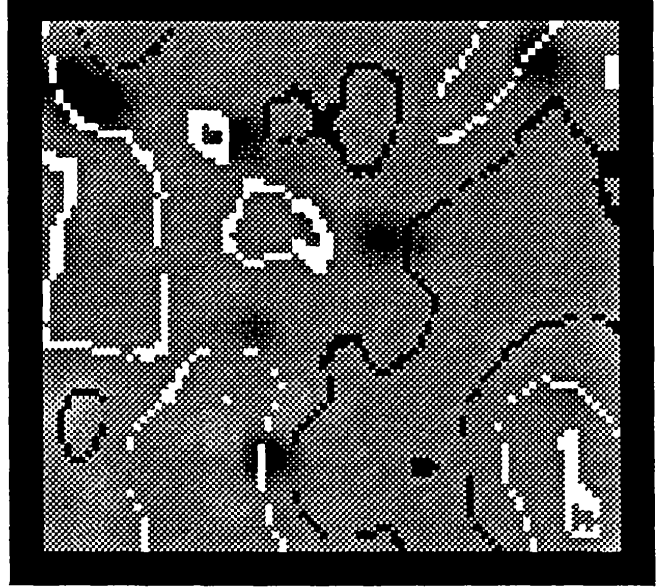

1948

Fig. 9. Two examples showing the magnetic fields associated with the growing and decaying supergranule cells. Data were obtained on May 11, 1986, UT time is given below in each frame. (a) A growing cell. (b) A decaying cell. White contours are receding motion, and black, approaching. Solar limb is toward the right of the image. Magnetic elements are represented by the gray scale map. The scale is the same as marked in Figure 8(a).

\section{Acknowledgements}

We wish to express our appreciations to staff at Big Bear Solar Observatory and National Solar Observatory/Kitt Peak for their support during observation runs. We are grateful to S. Martin, D. Gary, J. Kaufman, and F. Tang for reading the manuscript and giving helpful comments and suggestions. This work is supported by NSF under grant ATM-8513577 and NASA under grant NGL 05002034.

\section{References}

Antia, H. M., Chitre, S. M., and Pandey, S. K.: 1981, Solar Phys. 70, 67.

Chou, D., LaBonte, B. J., Braun, D. C., and Duvall, T. L.: 1989, in preparation. 
Deubner, F. L.: 1971, Solar Phys. 17, 6.

Duvall, T. L.: 1980, Solar Phys. 66, 213.

Frazier, E. N.: 1970, Solar Phys. 14, 89.

Giovanelli, G. R.: 1980, Solar Phys. 67, 211.

Hart, A. B.: 1954, Monthly Notices Roy. Astron. Soc. 114, 2.

Hart, A. B.: 1956, Monthly Notices Roy. Astron. Soc. 116, 38.

Janssens, T. J.: 1970, Solar Phys. 11, 222.

Kubičela, A.: 1976, Solar Phys. 47, 551.

Leighton, R. B.: 1957, Publ. Astron. Soc. Pacific 69, 497.

Leighton, R. B.: 1964, Astrophys. J. 140, 1547.

Leighton, R. B.: 1969, Astrophys. J. 156, 1.

Leighton, R. B., Noyes, R. W., and Simon, G. W.: 1962, Astrophys. J. 135, 474.

Livingston, W. C. and Orrall, F. Q.: 1974, Solar Phys. 39, 301.

Livingston, W. C., Harvey, J., Pierce, A. K., Schrage, D., Gillespie, B., Simmons, J., and Slaughter, C.: 1976a, Applied Opt. 15, 33.

Livingston, W. C., Harvey, J., Slaughter, C., and Trumbo, D.: 1976b, Applied Opt. 15, 40.

Martin, S. F.: 1988, Solar Phys. 117, 231.

Mosher, J.: 1976a, BBSO Preprint No. 159.

Mosher, J.: 1976b, Ph.D. Thesis, California Institute of Technology.

Musman, S. and Rust, D. M.: 1970, Solar Phys. 13, 261.

November, L. J., Toomre, J., and Gebbie, K. B.: 1981, Astrophys. J. 245, L123.

November, L. J., Toomre, J., Gebbie, K. B., and Simon, G. W.: 1982, Astrophys. J. 258, 846.

Rogers, E. H.: 1970, Solar Phys. 13, 57.

Shi, Z., Wang, J., and Patterson, A.: 1986, BBSO Preprint No. 257.

Skumanich, A., Smythe, C., and Frazier, E. N.: 1975, Astrophys. J. 200, 747.

Simon, G. W. and Leighton, R. B.: 1964, Astrophys. J. 140, 1120.

Smithson, R. C.: 1973, Solar Phys. 29, 365.

Stenflo, J. O., Harvey, J. W., Brault, J. W., and Solanki, S.: 1984, Astron. Astrophys. 131, 333.

Wang, H.: 1988, Solar Phys. 116, 1.

Wang, H. and Zirin, H.: 1988, Solar Phys. 115, 205.

Worden, S. P. and Simon, G. W.: 1976, Solar Phys. 46, 73.

Zirin, H.: 1985, Australian J. Phys. 38, 961.

Zirin, H.: 1987, Solar Phys. 110, 101.

Zirin, H.: 1989 , in preparation.

Zirin, H. and Wang, H.: 1987, Presentation in AAS Solar Physics Division Meeting, May 13-15, 1987, Honolulu. 